\title{
High fidelity full sized human patient simulation manikins: Effects on decision making skills of nursing students
}

\author{
Jennifer C F Loke', Bryant K Lee², Asmah Mohd Noor ${ }^{3}$, Susan Loh ${ }^{3}$ \\ 1. Faculty of Health and Social Care, University of Hull, UK. 2. Hymers College, UK. 3. School of Health Sciences, Nanyang \\ Polytechnic, Singapore. \\ Correspondence: Jennifer C F Loke. Address: Faculty of Health and Social Care, University of Hull, UK. Email: \\ j.loke@hull.ac.uk
}

Received: February 17, 2014

Accepted: March 24, 2014

Online Published: April 20, 2014

DOI : $10.5430 /$ jnep.v4n7p31

URL: http://dx.doi.org/10.5430/jnep.v4n7p31

\section{Abstract}

Background: The continued use of high fidelity full sized human patient simulation manikins (HF-HPSMs) for developing decision making skills of nursing students has led to growing research focusing its value on student learning and decision making skills.

Methods: In October 2012, a cross-sectional survey using the 24-item Nurse Decision-Making Instrument was used to explore the decision making process of 232 pre-registration nursing students (age $22.0 \pm 5.4$; 83.2\% female) in Singapore.

Results: The independent samples $t$-tests demonstrated three significant predictive indicators. These indicators include: prior experience in high fidelity simulation based on pre-enrolled nursing course ( $t=70.6, p=.001)$, actual hands-on practice $(t=69.66, p<.005)$ and active participation in debrief $(t=70.11, p<.005)$. A complete experience based on role-playing followed by active discussion in debrief was a significant contributor to the decision making process $(t=$ 73.6667, $p<.005)$. However, the regression model indicated active participation in debrief as a significant variable which explained its development $(t=12.633, p<.005)$.

Conclusions: This study demonstrated the usefulness of active participation in simulation learning for an analyticintuitive approach to decision making, however active participation in debrief was a more important influencing element than role-playing. In situations where resources are limited for students to experience hands-on role-playing, peer reviewing and feedback on others' experiences could benefit students, just as much. However, further study is warranted to determine the development of HF-HPSMs as a pedagogic tool for enhancing the decision making process of nursing students.

\section{Key words}

Critical thinking, Decision making skills, High fidelity full-sized human patient simulation manikins, Nursing students, Nursing education, Singapore 


\section{Introduction}

It is widely accepted that clinical reasoning in the decision making process is an essential component of nursing practice ${ }^{[1]}$. While the process by expert nurses involved both rational and intuitive thinking ${ }^{[2]}$, the process in stressful situations is believed to involve oscillations between analytic and intuitive reasoning ${ }^{[3]}$. Under the pressure to produce students who are prepared for effective clinical practice, there is an increasing demand to develop students' ability to engage in the kind of decision making process similar to that employed by qualified nurses at the point of their qualifying as registered nurses ${ }^{[4,5]}$. The development of decision making based on critical thinking skills has become important in pre-registration nursing education ${ }^{[6]}$; also known as pre-licensure or pre-qualifying nursing education. Consequently, innovative and challenging pedagogic strategies are being continuously explored to achieve the intended outcome. Human patient simulation is one such pedagogy.

The endeavour has resulted in a large amount of investment in healthcare education. The range of low-tech simulators to medium-tech simulation learning tools which had long been used in nursing education were gradually supplemented with more advanced technological simulators to provide virtual reality of Haptic systems ${ }^{[7]}$. From the early 2000s, full-scale high fidelity simulations using full body-sized human patient manikins (HF-HPSMs) based on sophisticated technologies have been extensively used. Due to the fact that HF-HPSMs could be programmed and used with actual medical equipment and supplies to provide physiologic responses to participants' actions in a realistic environment ${ }^{[7]}$, its use as a pedagogic tool had expanded exponentially in many parts of the world.

\subsection{Background}

The schools for pre-registration nursing education in Singapore were amongst the early adopters and intensive users of HF-HPSMs by Medical Education Technologies Incorporated $\left(\right.$ METI $\left.^{\circledR}\right)$ and SimMan ${ }^{\mathrm{TM}}$ by Laerdal ${ }^{\mathrm{TM}}$. This state of the art advanced technological approach in employing HF-HPSMs was frequently used alongside existing low to medium-tech simulators to consolidate students' clinical skill and experience. However, due to time constraints and limited resources against the sheer volume of pre-registration nursing students, unlike the use of low and medium-tech simulators, students had to take turns role-playing in HF-HPSMs sessions. Consequently, while some students had minimal hands-on experience, others remained audience members. In other words, not all would have equal opportunity for hands-on exercise experience. Also, due to time constraints, albeit addressing the entire group, lecturers would end up focusing on students who had the hands-on experience for self-reflection and actively engaging them in debrief. In essence, while only a few audience members could join in at various stages of discussions, many ended up listening to the discussion without any active contributions.

In various forms and degree of exposure to HF-HPSMs, all students would have received an equal amount of reflective debriefing sessions. Such practices in using HF-HPSMs for learning are ongoing throughout the entire educational experience, with an aim that each and every student would have participated in more than two role-play exercises and discussion in debrief by the time they reached the final year of the nursing programme. Resource constraint in this educational provision had given rise to disparity in exposure to HF-HPSMs during the course of the programme. It was therefore critical to gain knowledge of the effects attributed to the varying experience of HF-HPSMs on students' decision making abilities. As such, decision for further financial investment and effort could be made in creating identical student exposures to role-playing exercises at any one point in their curricular experience.

\subsection{Literature review}

The use of HF-HPSMs was popularised with the intention to develop effective clinical decision making skills based on competence in multiple dimensions including communication, prioritisation, interdisciplinary team working, technical skills and critical thinking ${ }^{[8]}$. However, since popularisation, research studies had focused primarily on the measurement of outcomes such as knowledge gain ${ }^{[9-11]}$, self-efficacy, self-confidence and skills attainment ${ }^{[12-18]}$. Studies which compared HF-HPSMs with other popular pedagogic approaches yielded some interesting results; demonstrating the superiority of HF-HPSMs in the student-centred approach to acquiring competent skills over both problem based 
learning ${ }^{[19]}$ and interactive case study ${ }^{[20]}$. Nevertheless, it was these positive findings that had added impetus to the development of HF-HPSMs in nursing education to them being widely used for developing decision making skills of the expert nurses; which in Benner's term was based on an intuitive-humanist approach ${ }^{[2]}$.

Subsequently, there was a gradual shift in research focus. As evidenced, more studies began to use critical thinking skills interchangeably with the decision making process to evaluate the effects of HF-HPSMs ${ }^{[21-24]}$. While that was the case, many other research studies concentrated on the development of critical thinking skills ${ }^{[25-28]}$. Due to the absence of a tool which could be consistently accepted to measure the concept of critical thinking ${ }^{[25]}$, such research studies were heterogeneous in their methodological approaches. Nevertheless, useful insights to the ways HF-HPSMs for learning could be enhanced was gleaned. For example, one study by Endacott et al. ${ }^{[29]}$ to evaluate final year students' performance in picking cues for action found that self-appraisal of performance was essential for making HF-HPSMs effective. Still, none of these studies were conclusive about the value of HF-HPSMs on decision making process of nursing students. As a result, studies are still being conducted to compare the elements of simulation learning to indicate its relation to decision making skills ${ }^{[30]}$. Yet, large amount of investment is continuously made for HF-HPSMs in nursing education based on its assumed value in developing students' decision making skills. Even if this was the case, how does HF-HPSMs as a pedagogic tool help in developing critical decision making skills amongst pre-registration nursing students? The objective of this study was to fill the research gap in determining the effects of HF-HPSMs on learners' decision making process.

\section{Research design}

A descriptive cross-sectional survey design was therefore used in October 2012. The tool was a Nurse Decision-Making Instrument, based on the continuum cognitive theory. This study was to capture the capability of HF-HPSMs in preparing students for complex decision making, which reflected that of the expert nurses'; a process which involved more than systematic rational thinking, but oscillated with intuitive reasoning to anticipate patients' need responsively.

\subsection{Method}

In order to establish the effects of HF-HPSMs on decision making skills, the survey was conducted when students had experienced HF-HPSMs.

\subsection{Setting}

The study was conducted in one of the main nursing educational providers in Singapore. Due to limitations in resources, students were able to receive identical amounts of exposure to low and medium-tech simulation learning, but not for HF-HPSMs experience. The former were 3 hours long, designed to teach and enhance new clinical skills, for example nasopharyngeal and endotracheal suctioning. In contrast; HF-HPSMs sessions were 60 minutes long and were conducted in a purpose-built high fidelity simulation lab. These HF-HPSMs sessions were designed to follow up the specific clinical skills previously acquired in low-tech and medium tech simulation to learn about managing patient care. Using the earlier example of learning the clinical skills for nasopharyngeal and endotracheal suctioning, the HF-HPSMs session would focus on developing skills and knowledge in caring for a post-operative patient who had a tracheostomy insertion. Typical examples of the scenarios are in Table 1.

\subsection{Participants}

All year-2 students $(n=233)$ who were in the second semester of a pre-registration diploma nursing programme were invited to participate in the study. At the time of this research, the students had already attended 4 HF-HPSMs sessions in their clinical skill groups, each of which comprised a maximum of 20 individuals. One week prior to each session, students received a brief outline of the case scenario for preparation based on self-directed learning. Depending on the scenario by which the information was presented to reflect a realistic clinical situation, 5 to 6 students were required to volunteer for a 30-minute team simulation exercise (role-play). 
Table 1. Examples of the simulation based learning scenarios for year two students

Care of a Patient with Chronic Obstructive Pulmonary Disease

A 60 year old truck driver complained of difficulty in breathing, chest tightness, cough and productive whitish sputum. He was seen by the doctor at the emergency department and admitted to your ward. He has history of diabetes for the past 10 years. He is on subcutaneous Insulatard insulin 16 units every morning. No known drug allergy. He has been smoking 10 cigarettes per day from the age of 22. Patient finds it hard to stop smoking.

Height: 1.6 m, Weight: 75 kg
Care of a Patient with Gastrointestinal bleeding

A 45 year old businessman is admitted to your ward complaining of the passage of black stools for 3 days, associated light-headedness and epigastric pain. He also relates that he cannot keep up with his usual work schedule because of fatigability. Upon further questioning he states that his stools are not only black, but are sticky and malodorous. He takes one aspirin (75 mg) per day for cardiac prophylaxis. Height: $1.56 \mathrm{~m}$ Weight: $60 \mathrm{~kg}$

Hence all students were expected to attend the sessions, and those involved in the role play were required to be actively thinking during the simulation exercise; to process and act on the information and to respond to new information presented as patients' responses. The role-playing by the few volunteers automatically land the remaining students as audience, who were expected to reflect on learning from peers' performance. The reflection was based on a list of expected learning outcomes as stated in the case scenario. In every HF-HPSMs session, 30 minutes were set aside for debriefing, which doubled as formative assessment of student learning. During each debrief, the nurse teacher would first seek discussion with the volunteers and then with the audience. The intention was to allow volunteers to self-assess and to create an opportunity for the audience to assess and critique the performance. In the critique, students were challenged to outline the common clinical manifestations and the appropriateness of the decision by peers in managing the clinical situation or patient conditions. In essence, unlike the low and medium-tech simulation learning, students' experience in each HF-HPSM varied and could take any of the following forms:

1) role-play (hands-on exercise) and debrief (engaged in discussion);

2) role-play (hands-on exercise) only;

3) debrief (engaged in discussion) only; and

4) none (as audience for both).

\subsection{Ethical considerations}

Ethics approval for this study as part of a larger study was obtained from the ethics committee of the faculty of the first author. It was later obtained from the ethics review board from the institution. All participants were informed of the aim of the study and had returned the questionnaires that indicated informed consent. Researchers' contact details were given to the participants for any queries.

\subsection{Data collection}

\subsubsection{Procedure}

Data collection took place in October 2012 in the second semester of the academic year. The questionnaires to establish information on students' demographic profile and decision making process were distributed by the third and fourth authors in the institution. This was conducted immediately after the fourth HF-HPSMs sessions, which took place at 2-week intervals. Students were given time to answer the questionnaires in lecture theatres and had returned the questionnaires immediately thereafter.

\subsubsection{I nstrument}

Data on students' decision making process were based on a 24-item Nursing Decision Making Instrument developed by Lauri and Salenterä ${ }^{[31]}$ whose permission for use was obtained via email. The instrument was modified from their original 54-item tool based on Hammond's mode ${ }^{[3]}$ of diagnostic judgement comprising 5 phases of clinical judgment including: 
1) a description of patient's state; 2) the collection of objective and subjective data for judgment; 3) the physician's and nurses' judgment; 4) integration of the judgment and; 5) making a hypothesis of solution. The modified instrument is a 5-point likert scale measuring users' nursing decision-making process as described in each statement. Participants ranked each statement ranging from 1-5 corresponding to options of "almost never", "rarely”, "sometimes”, "often”, and "almost always", respectively. While the scores to odd numbered items which described a systematic rational judgement task approach were reversed, those to even numbered items which described an intuitive approach remaining unchanged. The scores on decision-making process were then interpreted as follows:

1) < 67 points: analytically oriented

2) 68-78 points: oscillating between analytical and intuitive

3) 78 points: intuitively oriented

The instrument was developed through factor analysis of the original 54-item tool to ensure construct validity. Although the instrument has not been intensely validated, it has been used on 2095 nurses in 4 Norway hospitals and a Crobanch's alpha of 0.863 was established ${ }^{[32]}$. The Crobanch's alpha in the current study was 0.760 .

\subsection{Data analysis}

A total of 232 returned questionnaires were analysed, with only one being excluded due to an incomplete demographic section and many missing entries in the standardised tool. All data were entered into Statistical Packages for Social Sciences version 20 for Windows; computed and analysed with descriptive frequencies, independent sample $t$-tests and linear regression.

\section{Results}

\subsection{Demographic data and other independent variables}

The 232 student nurses; 193(83.2\%) females and 39 (16.8\%) males with ages ranging from 18-49 (mean 22.1; SD 5.4) participated in the study. Students held varying academic qualifications; 38 (16.4\%) held an enrolled nursing certificate and had clinical nursing experience (see Table 2).

Table 2. Demographic data of participants

\begin{tabular}{ll}
\hline Characteristics & $\mathbf{n}_{\mathbf{1 a}} \mathbf{( \% )}=\mathbf{2 3 2} \mathbf{( 1 0 0 \% )}$ \\
\hline Gender & $193(83.2 \%)$ \\
Female & $39(16.8 \%)$ \\
Male & $22.1(\mathrm{SD} 5.4)$ \\
Age (mean) & \\
Highest Qualifications & $2(0.9 \%)$ \\
Advanced Diploma (in non-Healthcare field) & $9(3.9 \%)$ \\
Diploma (in other fields) & $12(5.2 \%)$ \\
A level & $66(28.4 \%)$ \\
Pre-enrolled nursing certificate & $143(61.6 \%)$ \\
O level & \\
Years of experience as enrolled nurse & $194(83.6 \%)$ \\
0 & $9(3.9 \%)$ \\
$0-1$ year & $7(3 \%)$ \\
$1-2$ year & $6(2.6 \%)$ \\
$2-3$ year & $16(6.9 \%)$ \\
More than 3 year & \\
\hline
\end{tabular}


One hundred and forty eight students (63.8\%) had role-playing experience, and 117 of these had also contributed in debriefing sessions. This resulted in the majority of students $[n=171(73.7 \%)]$ having contributed in debrief sessions, and only $30(12.9 \%)$ as audience of others' learning without any experience in role-playing or discussion in debrief. As expected all students' experience in HF-HPSMs varied at the time of this study, some had participated in role-playing and debrief more often than others (see Table 3).

Table 3. Number of varying experience of students in HF-HPSMs sessions

\begin{tabular}{llllll}
\hline \multirow{2}{*}{ Number of students } & \multicolumn{6}{l}{ Number of the HF-HPSMs experiences } & & \\
\cline { 2 - 6 } & $\mathbf{4}$ & $\mathbf{3}$ & $\mathbf{2}$ & $\mathbf{1}$ & $\mathbf{0}$ \\
\hline role-playing & $6(2.6 \%)$ & $4(1.7 \%)$ & $31(13.4 \%)$ & $107(46.1 \%)$ & $84(36.2 \%)$ \\
actively participated in debrief & $9(3.9 \%)$ & $27(11.6 \%)$ & $50(21.6 \%)$ & $85(36.6 \%)$ & $61(26.3 \%)$ \\
\hline
\end{tabular}

\subsection{Students' scores in decision making skill tool}

The range in scores was 59-78 (mean 68.8; SD 3.8). The distribution of scores demonstrated that 74 (31.9\%) students were analytically oriented in decision making and 158 (68.1\%) students oscillated between an analytical and intuitive decision making process (see Figure 1).

\section{Types of decision process by students}
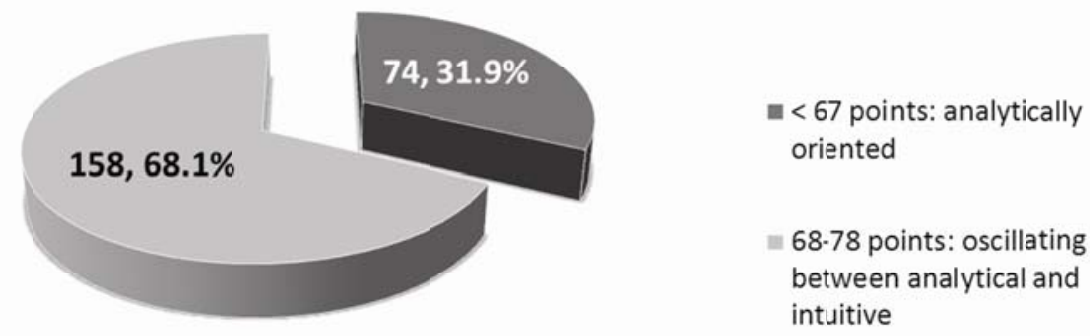

Figure 1. Distribution of scores by 232 students

The highest mean score (70.5; SD = 3.2) attributed to students who had role-play and discussion experience (see Table 4). High mean scores were similarly found amongst students who had participated in debrief alone (69.3; $S D=2.8$ ). While those who role-played scored a mean of 66.5 ( $S D=2.6)$, the audience for both activities scored the lowest mean (63.9; SD = 2.8) (see Table 4).

Table 4. Decision scores based on student experience

\begin{tabular}{llll}
\hline Experiences & Number of Students (\%) & Range of Scores & Mean Score (SD) \\
\hline Participated in role-play + debrief & $117(50.4 \%)$ & $60-78$ & $70.5(3.2)$ \\
Participated in role-play only & $31(13.4 \%)$ & $62-72$ & $66.5(2.6)$ \\
Participated in debrief only & $54(23.3 \%)$ & $64-78$ & $69.3(2.8)$ \\
Participated none (as audience) & $30(12.9 \%)$ & $59-70$ & $63.6(2.8)$ \\
\hline
\end{tabular}

When the decision-making skill scores were computed with $t$-tests and Pearson's $r$; a mean score of 70.06 was obtained by students who held a pre-enrolled nursing qualification, and this appeared to be statistically significant (see Table 5). When the other independent variables including the experience in 'role-play' and 'debrief' were analysed using independent sample $t$-tests, all 4 were significantly associated with students’ decision making process (see Table 5). 
Table 5. The association between variables and the decision making process

\begin{tabular}{|c|c|c|c|}
\hline Variables & Pearson $r$ & $t$-test mean & $\bar{P}$ \\
\hline \multicolumn{4}{|l|}{ Education } \\
\hline Others & & 68.28 & .001 \\
\hline Pre-enrolled nursing certificate & & 70.06 & \\
\hline Pre-enrolled nursing experience & 0.102 & & .121 \\
\hline \multicolumn{4}{|l|}{ HF-HPSMs participation } \\
\hline \multicolumn{4}{|l|}{ Active in role-playing } \\
\hline 1-4 times & & 69.66 & $<.005$ \\
\hline 0 time & & 67.26 & \\
\hline \multicolumn{4}{|l|}{ Active participation in debrief } \\
\hline 1-4 times & & 70.11 & $<.005$ \\
\hline 0 time & & 65.08 & \\
\hline \multicolumn{4}{|l|}{ Watching the exercise } \\
\hline 1-4 times & & 68.6593 & .001 \\
\hline 0 time & & 73.6667 & \\
\hline \multicolumn{4}{|l|}{ Watching the debrief session } \\
\hline 1-4 times & & 68.5919 & $<.005$ \\
\hline 0 time & & 73.6667 & \\
\hline
\end{tabular}

The analysis was continued using linear regression (see Table 6); variables which had significant statistical associations with the decision making process were analysed based on ANOVA statistics. The analysis demonstrated that having an enrolled nursing certificate had a lesser association with the decision making process than participation in HF-HPSMs session. Also 'participation in debrief' $(t=12.63, p<.05)$ tended to have a higher association than 'role-playing alone' $(t$ $=4.87, p<.05)$.

Table 6. Amount of variance in decision making process explained by the independent variables

\begin{tabular}{llll}
\hline Variables & Beta & $\boldsymbol{t}$ & $\boldsymbol{P}$ \\
\hline Education & & & .282 \\
Pre-enrolled nursing certificate & 0.052 & 1.007 & $<.005$ \\
HF-HPSMs participation & & & $<.005$ \\
Participated in role-playing & 0.239 & 12.633 & $<.005$ \\
\hline Participated in debrief & 0.606 & &
\end{tabular}

Note. $F=78.358, P<.005, \mathrm{R}^{2}=0.508$

\section{Discussion}

The population of nursing students in this study reflected the general profile in nursing where females are larger in number. The mean age of students was 22, reflecting the nursing students' profile in the country; students were generally of young age and were pursuing nursing as their first career. Also, indicated in the sample was a considerable number of students (n = 66) who had completed a pre-enrolled nursing programme with a local vocational institution to gain registration with the nursing regulatory board as enrolled nurses. This reflected a local phenomenon in which individuals who did not meet the criteria for pre-registration nursing courses would undertake pre-enrolled nursing courses. Having obtained an enrolled nursing certificate, many would then start working as enrolled nurses for at least a year before accessing pre-registration nursing education based on prior learning and experience. Those who met entry requirement post qualifying as enrolled nurses would seek entry into pre-registration nursing education without delay. As evidenced in the current study, of the 66 students who were qualified enrolled nurses, 48 had clinical experience in the capacity of enrolled nurses prior to undertaking the diploma nursing programme. 
In the exploration of the value of HF-HPSMs sessions based on students' self-reporting of their decision making process, its association with the demographic and background variables as well as the various experience of HF-HPSMs were established. Based on the scores, majority of students (69\%) tended to reflect the most commonly used model of cognition by trained nurses; which Hammond ${ }^{[3]}$ suggested involved oscillation between analytical and intuitive modes. These findings were consistent with that of Endacott et al. ${ }^{[29]}$, in which students were found to have used a combination of hypothetico-deductive reasoning and gut feelings. However, the authors ${ }^{[29]}$ attributed such behaviours to students' clinical inexperience which helped to explain the phenomenon of students missing significant cues which were sometimes, obvious. What was important as demonstrated in the current study was the beneficial effects of HF-HPSMs on the development of decision making skills. This finding was further demonstrated in the independent $t$-tests on the various demographic and other background variables. In the analysis the enrolled nursing qualification was found to have significant association with students' decision making process along with the actual HF-HPSMs experiences. This might be because of the typical HF-HPSMs experience these students received while undergoing the 2-year pre-enrolled nursing training in a vocational institution; where HF-HPSMs were intensively used for acquiring clinical competence and patient management skills. All of which were with the intention to develop students into effective assistant nurses. This explained why pre-enrolled nursing education exposure was demonstrated to be significantly associated with the decision process (70.06, $p=.001)$ and yet not a significant predictive indicator in the regression analysis $(t=1.007 ; p=.282)$.

In terms of HF-HPSMs sessions, the limited resources in the school had created 4 distinct learning experiences as described in section 3.1. The highest scores which reflected more of an analytical-intuitive mode of decision making process were obtained by students who had more experience in both role-play and discussion in debrief (mean $=70.5$ ). These findings were reflected in previous studies which unanimously demonstrated the positive outcomes of increased HF-HPSMs exposure on critical thinking skills ${ }^{[21-24]}$. The lowest scoring which reflected an analytical skill tended to be attributed to the lack of the two experiences (mean $=63.6$ ). This might be an expected outcome which helped to clarify the assumptions about the usefulness of HF-HPSMs in nursing education. A more interesting observation made in this study was found amongst students with different experiences; those who role-played alone tended to score less (mean $=66.5$ ) than those who solely engaged in discussions during debrief without role-playing experience (mean $=69.3$ ). These results indicate the value of debriefing over hands-on exercises in developing decision making skills.

This finding was consistent with previous work which indicated debrief as key to success in learning ${ }^{[33-35]}$. Hence, engaging students in reflective thinking post simulation exercise was paramount for learning. This was particularly so in institutions where the lack of resources had deprived students the equal opportunity to role-play for hands-on experience. In the case of the current institution with limited resources where students who role-played were without fail given the priority to contribute in debrief, it would be worthwhile to consider encouraging active discussions with the audience first. In other words, teachers should divert attention to students who had participated as a member of the audience, before returning to the team of role players. This recommendation was not exaggerated as the independent $t$-test for active participation in debrief was significantly higher $(t=70.11, p<.005)$ than the other 3 activities. In fact, the recommendation was important because students who remained as audience without any active participation in both hands-on exercises or debrief scored significantly the lowest $(t=65.08, p<.005)$. This suggestion was further supported by the findings using the regression model (see Table 5) that active participation in debrief was a significant variable which explained the decision making process of the students $(t=12.633 ; p<.05)$.

\section{Limitations}

This study was undertaken in one single nursing school in Singapore and participants were from one cohort. In addition, the findings were related to specific programme exposure and were therefore likely to be a problem for generalisation. Also, the tool in this study had not been intensively validated. All these limitations lend the current study to serve as a reference for future work on the effects of HF-HPSMs on decision making processes. Further work using larger sample size is needed to explore the value of HF-HPSMs on students' decision making process. More work is also required in order to establish how the pedagogic tool could be best implemented to enhance the complex process of decision making. 


\section{Conclusion}

This cross-sectional survey study has given some insights to the value of HF-HPSMs for team exercises based on real clinical scenarios in developing students' decision making skills. The findings illustrated the strengths of this pedagogic tool in developing decision making skills which resembled a qualified nurse; that involved oscillation between analytical and intuitive thinking. The decision making process was enhanced by hands-on experience followed by peer review and timely feedback through debrief. However, this study suggested that in the absence of hands-on experience, active participation in debrief would suffice in enhancing the decision making process. The superiority of debrief over the actual hands-on exercises for decision making skills demonstrated in the current study supported previous work. This study indicated that encouraging students' active discussion on HF-HPSMs exercises would help their reflective learning and was paramount to the development of decision making skills at a standard expected amongst registered nurses. The great value of peer review post HF-HPSMs in this study indicated a strong need to ensure active student participation in peer review activities. This is particularly important, in situations where limited physical resources might prevent students an equal opportunity for hands-on role-playing exercises.

\section{Acknowledgements}

This work, as part of a large evaluative study is based on the Prof Sir Ron Cooke International Scholarship awarded to the first author by the Higher Education Academy. All authors wish to thank Dr Chan Lee Mun, CEO of Nanyang Polytechnic and Miss Wong Luan Wah, Nurse Director of the School of Health Sciences for their permission for the study. All authors are most grateful to Dr Kah Wai Lee for his contributions to concept development and statistical analysis. We also wish to thank Dr Peter Willmot, Miss Deborah Lim Kai Hui and Professor Kate Galvin for their comments on the drafts.

\section{References}

[1] Banning, M. Clinical reasoning and its application to nursing: Concepts and research studies. Nurse Education in Practice. 2008; 8(3): 177-183. PMid:17869587 http://dx.doi.org/10.1016/j.nepr.2007.06.004

[2] Benner, P., Tanner, C., Chesla, C. Expertise in nursing practice: Caring, ethics and clinical judgment. Springer, New York; 1996.

[3] Hammond, K. R. Human judgement and social policy. Irreducible uncertainty, inevitable error, unavoidable injustice. Oxford University Press, New York; 1996.

[4] Hicks, F., Merrit, S., Elstein, A. Critical thinking and clinical decision making in critical care nursing: a pilot study. Heart and Lung. 2003; 32 (3): 169-180. http://dx.doi.org/10.1016/S0147-9563(03)00038-4

[5] Gunnarrsson, B. M., Stomberg, M. W. Factors influencing decision making among ambulance nurses in emergency care situations. International Emergency Nursing. 2009; 17(2): 83-89. PMid:19341993 http://dx.doi.org/10.1016/j.ienj.2008.10.004

[6] Durham, C. F., Alden, K. R. Enhancing patient safety in nursing education through patient simulation in R. G. Hughes (Ed) Patient safety and quality: An evidence-based handbook for nurses. Agency for Healthcare Research and Quality. Rockville, MD. Chapter 51, 2008.

[7] Decker, S., Sportsman, S., Puetz, L., Billing, L. The evolution of simulation and its contribution to competency. Journal of Continuing Education in Nursing. 2008; 39(2): 74-80. PMid:18323144 http://dx.doi.org/10.3928/00220124-20080201-06

[8] Maran, N. J., Galvin, R. J. Low-to high-fidelity simulation: A continuum of medical education? Medical Education 37(Suppl.1). 2003; 22-18. PMid:14641635 http://dx.doi.org/10.1046/j.1365-2923.37.s1.9.x

[9] Brannan J., White A. \& Bezanson J. Simulator effects on cognitive skills and confidence levels. Journal of Nursing Education. 2008; 47(11): 495-500. PMid:19010047 http://dx.doi.org/10.3928/01484834-20081101-01

[10] Hoffman, R. O’Donnell, J., Kim Y. The effects of human patients' simulators on basic knowledge in critical care nursing with undergraduate senior baccalaureate nursing students. Simulations in Healthcare. 2007; 2(2): 110-114. PMid:19088615 http://dx.doi.org/10.1097/SIH.0b013e318033abb5

[11] Jefferies, P. Rizzolo, M. NLN/Laerdal project summary report, designing and implementing models for innovative use of simulation to teach nursing care of ill adults and children: A national multi-site study. National League for Nursing, New York, 2006.

[12] Alinier G., Hunt B., Gordon R., Harwood C. Effectiveness of intermediate-fidelity simulation training technology in undergraduate nursing education. Journal of Advanced Nursing. 2006; 54(3): 359-369. PMid:16629920

http://dx.doi.org/10.1111/j.1365-2648.2006.03810.x

Published by Sciedu Press 
[13] Birch L., Jones N., Doyle P.M., Green P., McLaughlin A., Champney C., et al. Obstetric skills drills: evaluation of teaching methods. Nurse Education Today. 2007; 27(8): 915-922. PMid:17376563 http://dx.doi.org/10.1016/j.nedt.2007.01.006

[14] Childs, J., \& Seeples, S. Clinical teaching by simulation: Lessons learned from a complex patient care scenario. Nursing Education Perspectives. 2006; 27(3): 154-158.

[15] Gates, M., Parr, M., \& Hughen, J. Enhancing nursing knowledge using high-fidelity simulation. Journal of Nursing Education. 2012; 51(1): 9-15. PMid:22085206 http://dx.doi.org/10.3928/01484834-20111116-01

[16] Robinson, B. K., Dearmon, V. Evidence-based nursing education: Effective use of instructional design and simulated learning environments to enhance knowledge transfer in undergraduate nursing students. Journal of Professional Nursing. 2013; 29(4): 203-209. PMid:23910921 http://dx.doi.org/10.1016/j.profnurs.2012.04.022

[17] Radhakrishnan, K., Roche, J., Cunningham, H. Measuring clinical practice parameters with human patient simulator: A pilot study. International Journal of Nursing Education Scholarship. 2007; 4(1): 1-11. PMid:17402934 http://dx.doi.org/10.2202/1548-923X.1307

[18] Ruggenberg S. The Effect of Simulated Clinical Experience on Knowledge, Near Transfer, and Far Transfer in Nursing Education. EdD thesis, University of San Francisco, San Francisco, CA, 2008.

[19] Gers, M. A., Niemer, L. Utilization of problem based learning and simulation to promote integrated decision making in baccalaureate Nursing program. Clinical Simulation in Nursing. 2009; 5(3): e139. http://dx.doi.org/10.1016/j.ecns.2009.04.035

[20] Howard V.M. A Comparison of Educational Strategies for the Acquisition of Medical-Surgical Nursing Knowledge and Critical Thinking Skills: Human Patient Simulator vs. The Interactive Case Study Approach. EdD thesis, University of Pittsburgh, Pittsburgh, PA, 2007.

[21] Cant, R., Cooper, S. Simulation-based learning in nurse education: systematic review. Journal of Advanced Nursing. 2010; 66(1): 3-15. http://dx.doi.org/10.1111/j.1365-2648.2009.05240.x

[22] Chiang, V. C. L., Chan, S. S. C. An evaluation of advanced simulation in nursing: A mixed-method study. Collegian, 2013. http://dx.doi.org/10.106/j.colegn.2013.05.003.

[23] Ravert P.K.M. Patient Simulator sessions and Critical Thinking. Journal of Nursing Education. 2008; 47(12): 557-562. http://dx.doi.org/10.3928/01484834-20081201-06

[24] Schumacher, L. The impact of using high-fidelity computer simulation on critical thinking abilities and learning outcomes in undergraduate nursing students. Dissertation Abstracts International. 2004; 65(10b).

[25] Brown, D., Chronister, C. The effect of simulation learning on critical thinking and self-confidence when incorporated into an electrocardiogram Nursing Course. Clinical Simulation in Nursing. 2009; 5(1): e45-e52. http://dx.doi.org/10.1016/j.ecns.2008.11.001

[26] Garrett, B. M., MacPhee, M., Jackson, C. Implementing high-fidelity simulation in Canada: Reflections on 3 years of practice. Nurse Education Today. 2010; 31(7): 671-6. PMid:21075494 http://dx.doi.org/10.1016/j.nedt.2010.10.028

[27] Linden L.L. The effect of clinical simulation and traditional teaching versus traditional teaching alone on critical thinking of nursing students. EdD thesis, College of Saint Mary, Omaha, NE, 2007.

[28] Shinnick, M. A., Woo, M. A The Effect of human patient simulation on critical thinking and its predictors in prelicensure nursing students. Nurse Education Today. 2012. PMid:22564925

[29] Endacott, R., Scholes, J., Buykx, P., Cooper, S., Kinsman, L., McConnell-Henry. T. Final-year nursing students’ ability to assess, detect and act on clinical cues of deterioration in a simulated environment. Journal of Advanced Nursing. 2010; 66(12): 2722-2731. PMid:20735506 http://dx.doi.org/10.1111/j.1365-2648.2010.05417.x

[30] Galloway, S. J. Simulation Techniques to bridge the gap between novice and competent healthcare professionals. The Online Journal of Issues in Nursing. 2009; 14(2).

[31] Lauri, S., Salenterä. S. Developing an instrument to measure and describe clinical decision making in different nursing fields. Journal of Professional Nursing. 2002; 18(2): 93-100. PMid:11977007 http://dx.doi.org/10.1053/jpnu.2002.32344

[32] Bjørk, I. T. \& Hamilton, G. A. Clinical decision making of nurses working in hospital settings. Nursing Research and Practice. 2011. PMid:21994830 http://dx.doi.org/10.1155/2011/524918

[33] Cantrell, M. A. The importance of debriefing in clinical simulations. Clinical Simulation in Nursing. 2008; 4(2): e19-e23. http://dx.doi.org/10.1016/j.ecns.2008.06.006

[34] Fanning, R. M. \& Gaba, D. M. The role of debriefing in simulation-based learning. Simulation in Healthcare. 2007; 2(2): 115-125. PMid:19088616 http://dx.doi.org/10.1097/SIH.0b013e3180315539

[35] Issenberg S.B., McGaghie W.C., Petrusa E.R., Gordon D.L., Scalese, R.J. Features and uses of high-fidelity medical simulations that lead to effective learning: A BEME systematic review. Medical Teacher. 2005; 27(1): 10-28. PMid:16147767 http://dx.doi.org/10.1080/01421590500046924 